\title{
ROS/KRAS/AMPK signaling determines gemcitabine-induced pancreatic cancer stem-like cell property in vitro
}

\author{
Hengqiang Zhao ${ }^{1}$, Hehe Li ${ }^{1}$, Qingke Duan ${ }^{1}$, Zhengle Zhang ${ }^{1}$, Heshui Wu${ }^{1}$, Qiang \\ Shen ${ }^{2}$, Chunyou Wang ${ }^{1}$ and Tao Yin ${ }^{1}$ \\ ${ }^{1}$ Department of Pancreatic Surgery, Union Hospital, Tongji Medical College, Huazhong University of Science and Technology, \\ Wuhan 430022, China \\ ${ }^{2}$ Department of Clinical Cancer Prevention, The University of Texas MD Anderson Cancer Center, Houston, TX 77030, USA \\ Correspondence to: Tao Yin, email: ytwhun@126.com \\ Chunyou Wang, email: chunyouwang52@126.com
}

Keywords: ROS; KRAS; AMPK; glycolysis; cancer stem cells

Received: June 20, $2017 \quad$ Accepted: January 04, $2018 \quad$ Published: January 09, 2018

Copyright: Zhao et al. This is an open-access article distributed under the terms of the Creative Commons Attribution License 3.0 (CC BY 3.0), which permits unrestricted use, distribution, and reproduction in any medium, provided the original author and source are credited.

\section{ABSTRACT}

Poor prognosis of pancreatic cancer (PanCa) is partially due to chemoresistance to gemcitabine (GEM), the standard chemotherapy. Dysregulated glucose metabolism is revealed to contribute to therapeutic resistance and pluripotent state. Cancer stem cells (CSCs) rewire cellular metabolism to survive the chemotherapeutics and initiate cancer relapse. However, few studies focus on the effect of GEM on cancer cell metabolism, the stemness status, and the involved mechanisms which are crucial in PanCa treatment. In this study, we demonstrate that GEM treatment induces metabolic reprogramming from mitochondrial oxidation to glycolysis and promotes cancer stem-like status. However, inhibition of glycolysis using 2-deoxyD-glucose (2-DG) suppresses cancer stemness and strengthens the cytotoxicity of GEM. We further demonstrate that GEM-induced metabolic reprogramming is KRASdependent as knockdown of KRAS reverses the metabolic shift. In addition, GEMinduced metabolic reprogramming activates AMP-activated protein kinase (AMPK) which promotes glycolytic flux and cancer stemness. Our results further reveal the involvement of GEM-induced reactive oxygen species (ROS) in the activation of KRAS/ AMPK pathway in regulation of metabolic reprogramming and cancer stemness, while $\mathrm{N}$-acetyl-L-cysteine (NAC), a ROS scavenger, inhibits the activated KRAS/AMPK pathway, which is further validated by introducing exogenous hydrogen peroxide $\left(\mathrm{H}_{2} \mathrm{O}_{2}\right)$. Collectively, these findings reveal an undesired effect of GEM in PanCa treatment. Therefore, regulating cellular redox, targeting KRAS/AMPK signaling, or reversing metabolic reprogramming may be effective approaches to eliminate CSCs and enhance chemosensitivity of GEM to improve the prognosis of patients with PanCa.

\section{INTRODUCTION}

Cytotoxic chemotherapy with gemcitabine (GEM) continues to be the first-line treatment for pancreatic cancer (PanCa), however, minimal progress has been made to improve the prognosis of $\mathrm{PanCa}$ patients [1]. The limited effectiveness has been attributed to the existence of relatively rare, but highly chemoresistant cancer stem cells (CSCs) $[2,3]$. CSCs are reported to have inherently high tumorinitiating potential, which is implicated in tumor relapse as well as establishment of metastases $[4,5]$. Accumulating evidence has shown that the therapeutic resistance of cancer may result from the dysregulation in glucose metabolism [6]. Tumor cells rewired their metabolic pathways to ensure energy supply and maintain survival to escape external lethal insult [7]. In addition, ionizing radiation could induce metabolic alteration which was closely associated with CSC phenotype [8], and promote cancer stem-like cell population expansion and resistance to anticancer drugs [9]. Therefore, it is interesting to investigate the effect of GEM on cancer 
cell metabolism and stemness to illuminate the underlying mechanisms of chemoresistance in PanCa.

Oncogenic $K R A S$ mutation functions as a key driver in the initiation and maintenance in approximately $90 \%$ of PanCa [10]. KRAS oncogene encodes a small GTPase $(21 \mathrm{KDa})$ which is active in a GTP bound form and inactive when bound to GDP [10]. Accumulating evidence suggests that the altered metabolic switch is under the control of various oncogenic signals [11]. Aberrant activation of KRAS can cause dysfunction of oxidative phosphorylation (OXPHOS), leading to elevated glycolysis and tumor development [12]. The transition from OXPHOS into glycolysis is a prerequisite to fuel proficient reprogramming directing a differentiated cytotype back to the pluripotent state [13]. Such transition of cellular metabolism could cause energy crisis; to restore energy supply, AMP-activated protein kinase (AMPK), a key regulator of cellular energy metabolism exerts stimulatory effects on ATP generation via promoting glucose uptake and transport [14]. In addition to act as a stress-response molecule, AMPK is associated with drug resistance and CSCs enrichment [15].

Induction of reactive oxygen species (ROS) upon GEM treatment has been reported previously $[16,17]$. Although multiple studies have shown that high levels of ROS are cytotoxic while low levels of ROS may be tumor promoting $[17,18]$, alteration of ROS levels in human cohorts do not produce the desired effects observed in preclinical models. Exploration of the underlying signaling linked with ROS might provide more clues on cancer management.

In this study, we demonstrate that GEM treatment induces metabolic reprogramming toward glycolysis, leading to the induction of PanCa cell stem-like property and chemoresistance. Mechanistically, GEM-induced metabolic reprogramming and cancer stemness are regulated by reactive oxygen species (ROS)-mediated activation of KRAS/AMPK pathway. These findings may shed light on how GEM induces therapeutic resistance and provide rationale and windows for better managing $\mathrm{PanCa}$.

\section{RESULTS}

\section{GEM treatment induces metabolic reprogramming toward glycolysis in PanCa cells}

In our recent study, we demonstrated that GEMresistant PanCa cells underwent metabolic shift, becoming more glycolytic [19]. However, it remains unclear whether GEM treatment induces metabolic reprogramming. We first examined the effect of GEM on the expression of glycolytic genes. As revealed by qRT-PCR analysis, GEM treatment resulted in higher mRNA levels of the majority of glycolytic enzymes reported to contribute to the aggressiveness or drug resistance in cancer cells such as GLUT1, HK2, PFKP, ALDOA, TPI1, PGK1, PGAM1, PKM2, and LDHA in PANC-1, SW1990, and Patu8988 cells (Supplementary Figure 1A).
We then utilized (2-(N-(7-nitrobenz-2-oxa-1,3-diazol4-yl)amino)-2-deoxyglucose) (2-NBDG), a fluorescent deoxyglucose analog, to monitor glucose uptake and found that GEM treatment increased 2-NBDG uptake in PanCa cells (Figure 1A). To confirm the up-regulation of glycolysis at the functional level, we monitored the release of lactate in the extracellular medium. After GEM treatment, the lactate concentration in the medium was strongly augmented, indicating an increased pyruvate to lactate conversion (Figure 1B). Importantly, when 2-deoxy-D-glucose (2-DG), a glucose analog which competes with glucose for uptake via GLUT1, as well as being phosphorylated by HKII, allosterically inhibits HKII and thus functions as a glycolytic inhibitor [20], was introduced, the elevated lactate production induced by GEM was significantly reduced (Figure 1C). Likewise, GEM dose-dependently up-regulated the protein and mRNA expressions of glucose transporter 1 (GLUT1) which is responsible for the uptake of extracellular glucose, and lactate dehydrogenase A (LDHA), an enzyme responsible for the conversion of pyruvate into lactate (Figure 1D). Collectively, the high glucose uptake and lactate production rate suggested that the glycolysis flux was up-regulated upon GEM treatment.

At the intersection of aerobic glycolysis and respiration, pyruvate dehydrogenase kinase (PDK), which inhibits pyruvate dehydrogenase (PDH) thereby prevents the entry of pyruvate into OXPHOS-based metabolism, plays a key role in cellular glucose metabolism. Interesting, we detected four genes encoding kinase (PDK1, PDK2, PDK3 and PDK4) that inhibit the PDH enzyme complex activity and found that PDK2 gene was significantly induced at transcript level in all three tested cell lines with GEM exposure (Supplementary Figure 1B). We therefore examined the protein levels of PDK2. As expected, GEM dose-dependently up-regulated the expression of PDK2 as indicated by Western blot analysis (Figure 1E). Likewise, GEM clearly increased the phospho-PDHE1- $\alpha$ (Ser 293) (p-PDHE1- $\alpha$ ) in a dose-dependent manner (Figure 1E). PDK phosphorylates the PDHE1- $\alpha$ subunit and inactivates the PDH enzyme complex that converts pyruvate to acetylcoenzyme A, thereby inhibiting pyruvate metabolism via the tricarboxylic acid (TCA) cycle. In addition, mitochondrial membrane potential $(\Delta \Psi \mathrm{m})$ is generated by OXPHOS activity, thus, a decrease in $\Delta \Psi \mathrm{m}$ is indicative of decreased electron transport and OXPHOS activity [21]. We also observed that GEM treatment decreased $\triangle \Psi \mathrm{m}$ (Figure 1F) without affecting the cell viability (Supplementary Figure 2A). All these findings suggested that GEM treatment induced metabolic reprogramming.

\section{Inhibition of glycolysis abrogates GEM-induced enhancement of cancer stemness}

Transition from OXPHOS, typical of somatic cells, into glycolysis is a prerequisite to fuel proficient reprogramming directing a differentiated cytotype back 
to the pluripotent state [13]. We therefore investigated the cancer stem-like property of GEM-treated PanCa cells. CSCs are defined by their ability to form tumor spheres in non-adherent conditions [3, 22]. Three PanCa cells (PANC-1, SW1990, and Patu8988) were treated with GEM at a "harmless" dose (5 $\mu \mathrm{M}, 24 \mathrm{~h})$ (Supplementary Figure 2A) exhibited enhanced ability to form tumor spheres in ultra-low adhesion plates (Supplementary Figure 2B). Since the pluripotent markers Nanog and Sox2 are functionally involved in the maintenance of stemness [22], we determined expression of Nanog and Sox 2 and found increased mRNA and protein expressions in all three cell lines treated with GEM in a dose-dependent manner (Supplementary Figure 2C). We further found that GEM significantly increased gene expression of ALDH, OCT4, KLF4, CXCR4, and CD24 reported as CSC markers in all three cell lines [3, 23-25], and EPCAM in SW1990 (Supplementary Figure 2D). In addition, GEM up-regulated ABCG2 and MDR1 gene levels associated with drug resistance [26] (Supplementary Figure 2E). Additionally, ID-1, MUC-1 and MUC-4 were all up-regulated upon GEM treatment in pancreatic cancer cells, indicating the decreased
A
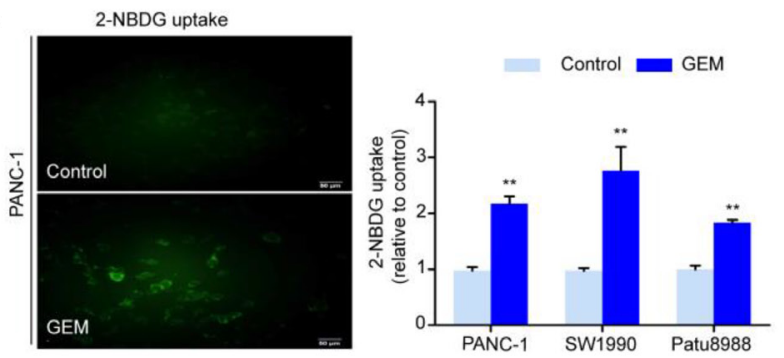

B

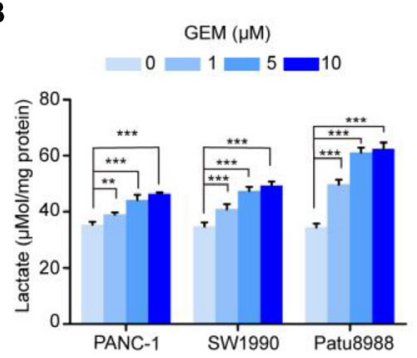

C

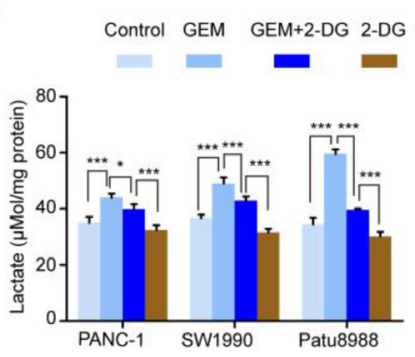

GEM ( $(\mu M)$

D
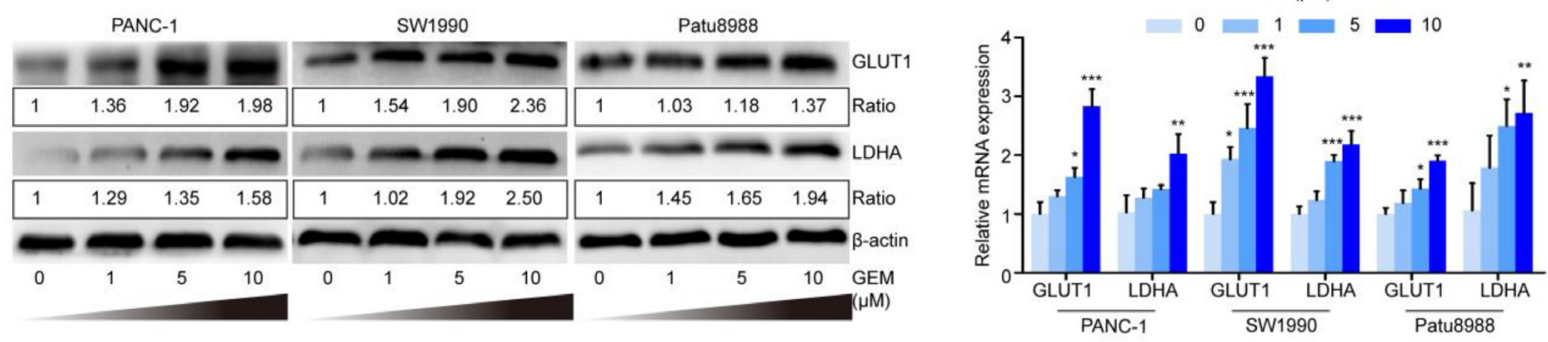

E

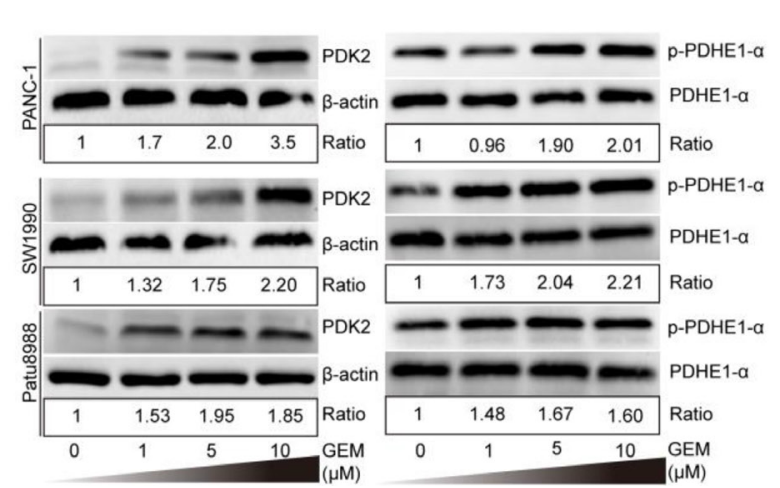

F
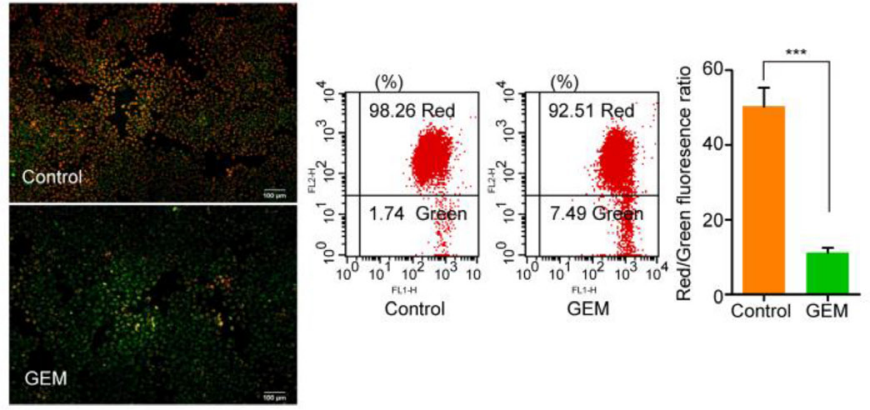

Figure 1: GEM induces metabolic reprogramming towards glycolysis in PanCa cells. (A) Glucose uptake was measured using 2-NBDG. PANC-1, SW1990, and Patu8988 cells were treated with GEM or not (5 $\mu \mathrm{M}, 24 \mathrm{~h})$. Representative images of 2-NBDG uptake in PANC-1 cells by fluorescent microscope (left) and quantification of the assay by flow cytometry (right). Scale bar, $50 \mu \mathrm{m}$. (B) Extracellular lactate release was measured in the cells following $36 \mathrm{~h}$ exposures to different doses of GEM. (C) Effect of 2-DG on GEM-induced lactate production. All the three cells were pretreated with 2-DG (5 mM, $1 \mathrm{~h}$ ) followed by treatment with GEM. (D) GEM dose-dependently up-regulated the expression of GLUT1 and LDHA in both protein and mRNA levels. All the three cell lines were treated with increasing concentrations of GEM for $24 \mathrm{~h}$. Total protein or RNA was extracted for Western blot or qRT-PCR analyses. Ratio is expressed as fold change compared with control values normalized to 1 after normalized by $\beta$-Actin. (E) Western blot analysis showed increasing expressions of PDK2 and p-PDHE1- $\alpha$. Cells were treated as indicated in (D). Ratio represents the intensity of band of PDK2 or p-PDHE1- $\alpha$ normalized $\beta$-Actin or total PDHE1- $\alpha$, respectively, and then normalized by control. Densitometry was performed by Image Lab software. (F) $\triangle \Psi \mathrm{m}$ in PANC-1 cells treated with GEM $(5 \mu \mathrm{M}, 24 \mathrm{~h})$ by JC-1 method. GEM treatment induced a depolarized $\Delta \Psi \mathrm{m}$ with more green than red JC-1 fluorescence. Representative images of fluorescence microscope (left), flow cytometry analysis (middle), and quantification (right) are shown. Scale bar, $100 \mu \mathrm{m}$. The data shown are from three independent experiments. Bars represent means \pm SD. $\beta$-Actin was served an internal control. ${ }^{*} P<0.05 ;{ }^{* *} P<0.01 ;{ }^{* * *} P<0.001$. GEM: gemcitabine. 
differentiated extent [27, 28] (Supplementary Figure 2F). Furthermore, GEM dose-dependently increased epithelialmesenchymal transition (EMT)-associated genes such as N-cadherin, Vimentin, Snail1, and Zeb1 in pancreatic cancer cells [29] (Supplementary Figure 2G).

We further investigated whether inhibition of glycolysis could abrogate GEM-induced cancer stemness in PanCa cells. We found that 2-DG, a glycolytic inhibitor, partially abrogated GEM-induced expression of pluripotent marker Nanog and Sox 2 at protein and mRNA levels (Figure 2A-2B). To further confirm that GEM-induced up-regulation of glycolysis promoted cancer stemness, we examined the expression of CD133, a CSC surface marker of PanCa [22, 25, 29, 30]. 2-DG also significantly decreased GEMinduced CD133 expression (Figure 2C-2D). Furthermore, treatment with 2-DG at a "harmless" dose (5 mM, $24 \mathrm{~h}$ ) to Patu8988 cells (Supplementary Figure 2A) suppressed their sphere formation ability (Supplementary Figure $2 \mathrm{H}$ ). Moreover, 2-DG significantly reinforced the cytotoxicity of GEM toward PanCa cells with a significant decrease in IC50 in PANC-1 and SW1990 cells (Figure 2E-2F). Therefore, inhibition of glycolysis suppressed GEM-induced stemness and enhanced therapeutic responses of GEM in PanCa cells.

\section{$K R A S$ oncogene is involved in GEM-induced metabolic reprogramming and cancer cell stemness}

It has been reported that $K R A S(\mathrm{G} 12 \mathrm{~V})$ transformation led to mitochondrial dysfunction and metabolic switch [12]. We therefore investigated the contributing role of KRAS in GEM-induced metabolic reprogramming. We first evaluated the effect of GEM on KRAS activation. As revealed in Figure 3A, GEM exposure effectively activated KRAS, showing a higher level of GTP-bound KRAS compared with the control group. We further assessed the effect of $K R A S$ extinction on metabolic reprogramming. Knockdown of $K R A S$ was first verified, which resulted in a decrease in the most of glycolytic genes (Supplementary Figure 3A-3B). Importantly, knockdown of $K R A S$ reduced lactate production (Figure 3B). Meanwhile, KRAS knockdown suppressed the expression of PDK2 and consequently decreased p-PDHE1- $\alpha$ (Figure 3C), indicating the restoration of OXPHOS. Additionally, the $\triangle \Psi \mathrm{m}$ was not decreased after KRAS knockdown as revealed by fluorescent microscope and flow cytometry analyses (Figure 3D). The maintenance of $\Delta \Psi \mathrm{m}$ indicates the functional OXPHOS. These results suggested that the activation of KRAS can inhibit the mitochondrial function and drive metabolic switch to glycolysis.

We further assessed the role of KRAS in cancer stemness maintenance and chemoresistance. KRAS knockdown decreased the sphere formation ability (Figure 3E) and the expression of pluripotent markers in both protein and mRNA levels (Figure 3F-3G). In addition, $K R A S$ knockdown significantly enhanced the cytotoxicity of GEM, 2-DG, or both with decreased IC50 (Figure 3H). Together, these results established that the metabolic switch from glycolysis to OXPHOS induced by KRAS knockdown inhibited cancer stemness and sensitized PanCa cells to chemotherapeutics.

\section{GEM-induced metabolic shift activates AMPK}

As observed above, GEM induced a metabolic shift from OXPHOS toward aerobic glycolysis which was a less efficient pathway for cellular energy supply. AMPK is an evolutionarily conserved energy sensor, modulating cellular energy flux in response to energy crisis [31]. We therefore examined the effect of GEM on AMPK activation. As expected, GEM dose-dependently increased the phosphorylation of AMPK $\alpha$ (Thr172) (pAMPK $\alpha)$ (Figure 4A), which is thought to correlate with the enzyme activity [32]. To further examine whether the rewired cellular metabolism accounted for AMPK activation, we examined the effect of KRAS knockdown on AMPK activation. As expected, knockdown of $K R A S$ resulted in a reduced pAMPK $\alpha$ expression (Figure 4B), suggesting a regulatory role of $K R A S$-mediated cellular metabolism in AMPK activation.

It is well documented that AMPK is involved in the regulation of glycolytic flux [33]. We therefore investigated whether AMPK was involved in GEMinduced metabolic reprogramming. We pretreated cancer cells with compound $\mathrm{C}$, an inhibitor of AMPK, followed by exposure to GEM. The results showed that GEMinduced $\mathrm{pAMPK} \alpha$ were abrogated after introducing compound $\mathrm{C}$ which showed marginal effect on $\mathrm{p}-\mathrm{PDHE} 1-\alpha$ (Figure 4C). However, compound $\mathrm{C}$ significantly inhibited GEM-induced lactate production (Figure 4D), suggesting the inhibition of glycolysis. We also observed that cancer cells treated with compound $\mathrm{C}$ exhibited reduced sphere formation (Figure 4E), diminished expression of pluripotent markers (Figure 4F). In addition, compound $\mathrm{C}$ strengthened the cytotoxicity of GEM toward PanCa cells, showing a decreased IC50 (Figure 4G and Figure 2F).

To further illuminate the potential role of AMPK in glycolytic flux, we introduced an AMPK-dependent activator A-769662. Treatment with A-769662 activated AMPK, while had no obvious effect on p-PDHE1- $\alpha$ (Supplementary Figure 4A). We did not observe difference in lactate production after A-769662 treatment (data not shown). However, A-769662 treatment promoted the glycolytic gene expression such as GLUT1 and LDHA (Supplementary Figure 4B). Contrary to compound C, A-769662 promoted sphere formation (Supplementary Figure 4C), and pluripotent marker expression (Supplementary Figure 4D), and tumor cell viability in appropriate concentrations (Supplementary Figure 4E). Collectively, these findings demonstrated that AMPK 
mediated GEM-induced up-regulation of glycolysis and cancer stemness induction.

\section{GEM-induced ROS participates in the activation of KRAS/AMPK pathway, metabolic reprogramming, and cancer stemness}

Induction of ROS upon GEM treatment has been reported previously $[16,17]$. To examine whether GEMinduced ROS activates the KRAS/AMPK pathway, we first examined ROS production upon GEM treatment. Consistent with previous findings, GEM treatment induced a dose-dependent increase in ROS levels which were abrogated after the introduction of N-Acetyl-cysteine (NAC) (a free radical scavenger) (Figure 5A). Importantly, GEM-induced up-regulation of KRAS-GTP and pAMPK $\alpha$ were both abrogated by pretreatment with NAC (Figure 5B). Next, we found that GEM-induced lactate production was reduced (Figure 5C), and p-PDHE1- $\alpha$ expression was diminished by NAC (Figure 5D), indicating that GEMinduced activation of KRAS/AMPK signaling was ROSdependent.

We further found that GEM-induced sphere formation (Figure 5E), CD133 expression (Figure 5F), and pluripotent markers (Figure 5G-5H) were all inhibited by pretreatment with NAC. To avoid ROS-induced oxidative damage, cancer cells often up-regulated their antioxidant defense capacity. We observed a significant increase in the expression of antioxidant genes such as superoxide dismutase 1 (SOD1), superoxide dismutase 2 (SOD2), catalase (CAT), glutathione peroxidase 1 (GPX1), and nuclear factor (erythroid-derived 2)-like 2 ( $\mathrm{Nrf} 2$ ) in PanCa cells (Supplementary Figure 5), suggesting that GEM induced oxidative stress accompanied with an increase of the antioxidant reactions.

To further establish a role of oxidative stress in the activation of KRAS/AMPK pathway, we introduced exogenous $\mathrm{H}_{2} \mathrm{O}_{2}$ to induce an increase in ROS levels comparable to that induced by GEM in PanCa cells (Figure 6A). Similar to GEM treatment, $\mathrm{H}_{2} \mathrm{O}_{2}$ induced KRAS/AMPK activation, which was also inhibited by NAC (Figure 6B). In addition, $\mathrm{H}_{2} \mathrm{O}_{2}$ increased glycolytic gene expression (Supplementary Figure 6), and lactate production (Figure 6C), indicating the elevation of glycolytic flux. Importantly, $\mathrm{H}_{2} \mathrm{O}_{2}$-induced lactate and p-PDHE1- $\alpha$ were both diminished with NAC treatment (Figure 6C-6D). Moreover, $\mathrm{H}_{2} \mathrm{O}_{2}$-induced sphere formation, $\mathrm{CD} 133$ expression, and pluripotent markers were all decreased by the introduction of NAC (Figure 6E-6H) . Collectively, these findings established that GEM induced metabolic reprogramming and cancer stemness via ROSdependent activation of KRAS/AMPK pathway (Figure 7).

\section{DISCUSSION}

GEM has been the standard of care chemotherapy for PanCa patients [1]. However, its therapeutic effect is limited and patients who do respond ultimately develop chemoresistance [34]. Accumulating evidence suggests that CSCs are resistant to conventional chemotherapy and radiotherapy, and are a major cause of cancer recurrence

B
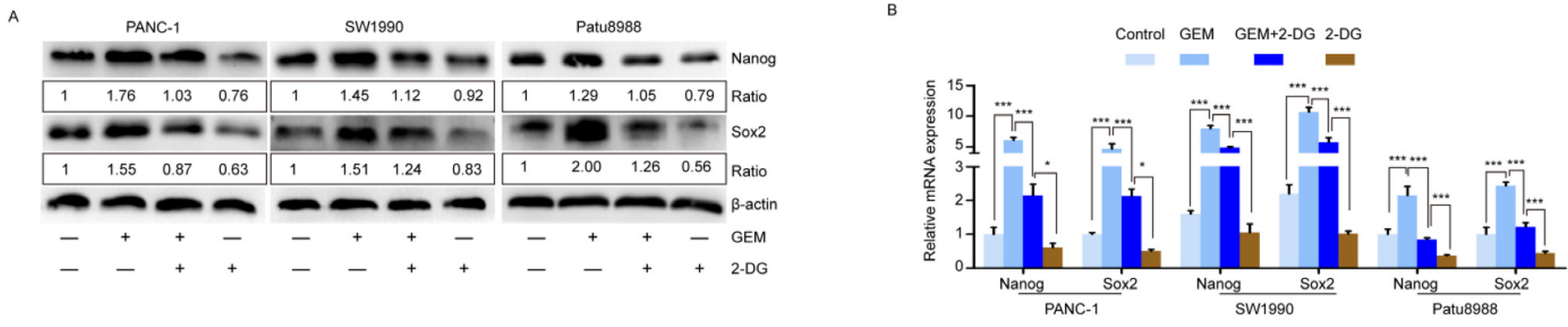

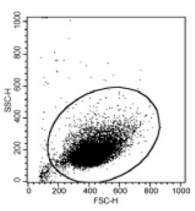

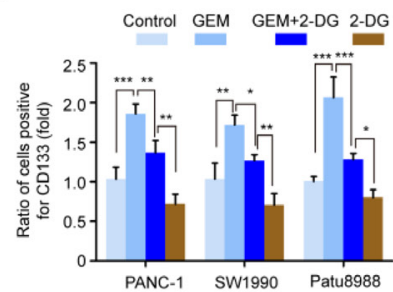

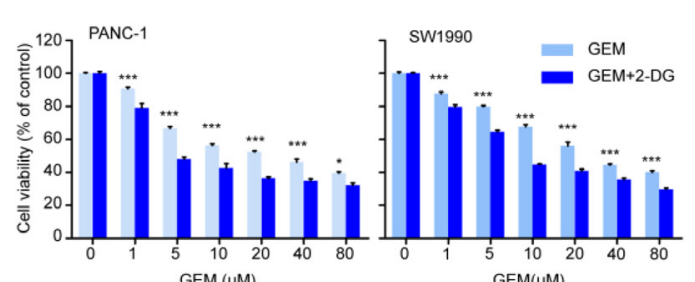

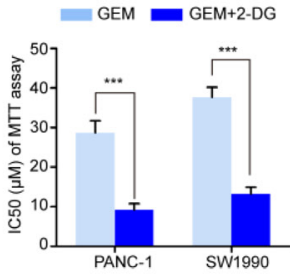

Figure 2: GEM-induced cancer cell stemness is abrogated via inhibition of glycolysis. (A-B) Inhibition of pluripotent markers Nanog and Sox2 with 2-DG treatment. PANC-1, SW1990, and Patu8988 cells were pretreated with 2-DG (5 mM, $1 \mathrm{~h})$ followed with GEM treatment for $24 \mathrm{~h}$. Total protein or RNA were extracted for Western blot or qRT-PCR analyses, respectively. Ratio is expressed as fold change compared with control values normalized to 1 after normalized by $\beta$-Actin. $\beta$-Actin was served as an internal control. (C-D) Flow cytometry analysis of CSC surface marker CD133. Representative images of the gating strategy of PANC-1 cells (C) and quantification of the assay (D). (E-F) PANC-1 and SW1990 cells were treated with increasing concentrations of GEM or combined with 2-DG (5 mM) for $48 \mathrm{~h}$. Cell viability was measured using MTT assay. Representative results (E) and quantification of the IC50 (F) are shown. All experiments were repeated at least 3 times. Bars represent means \pm SD. ${ }^{*} P<0.05 ;{ }^{* *} P<0.01 ;{ }^{* * *} P<0.001$. GEM: gemcitabine. 
and metastasis $[2,3,22,35,36]$. Therefore, illumination of the underlying mechanisms involved in chemoresistance and cancer stem-like property would shed light on identifying potential therapeutic windows to improve PanCa treatment. In the present study, we for the first time demonstrate that GEM treatment induces metabolic reprogramming toward glycolysis via ROS-mediated activation of KRAS/AMPK pathway, attributing to the enhanced stemness in PanCa cells in vitro.

Both induced pluripotent stem cells and embryonic stem cells undergo metabolic transition, including upregulation of glycolysis and down-regulation of OXPHOS [13]. Similar to these phenomena, we found that GEM induces metabolic reprogramming with enhanced
A

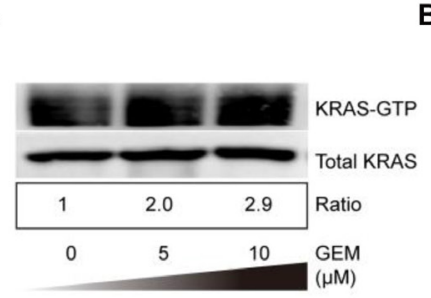

D

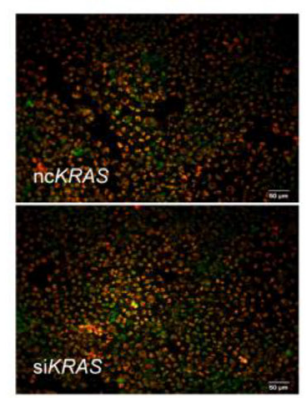

$\mathbf{F}$

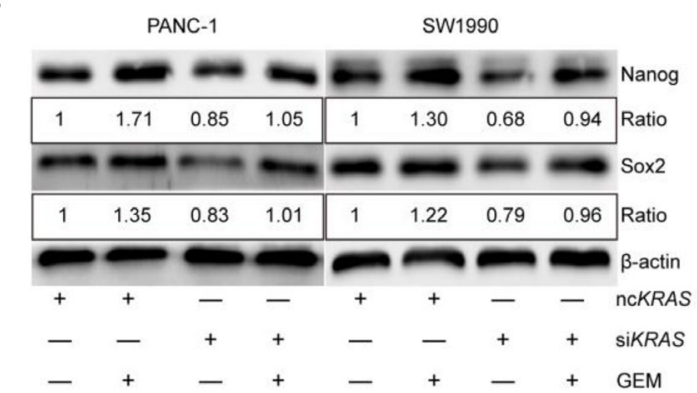

B

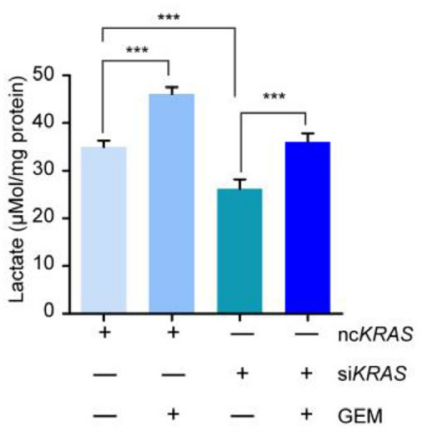

E
C

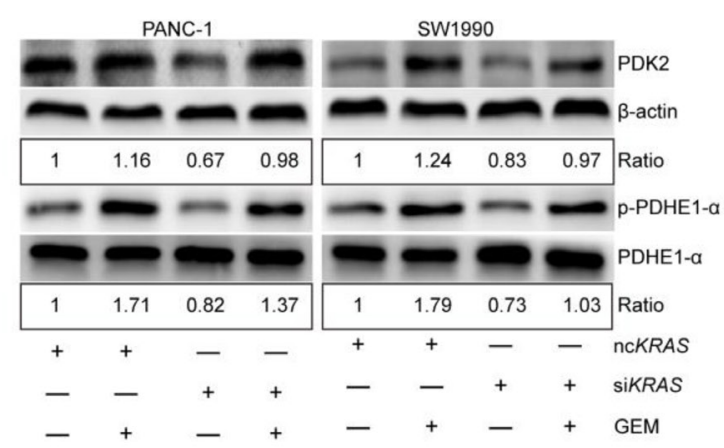

Figure 3: KRAS oncogene is involved in the regulation of GEM-induced metabolic reprogramming and cancer cell stemness. (A) Effect of GEM treatment on KRAS activation. PANC-1 cells were treated with GEM for $24 \mathrm{~h}$ and activation of KRAS was analyzed as described in the "Material and Method". Ratio represents the intensity of band of KRAS-GTP normalized total KRAS and then normalized by control. (B) Knockdown of KRAS inhibited lactate production in PANC-1 cells. Cells were first transfected with siRNA targeting $K R A S$ (siKRAS) or negative control siRNA (ncKRAS). Following $48 \mathrm{~h}$ of transfection, cells were treated with or wihout GEM $(5 \mu \mathrm{M})$ for the next $24 \mathrm{~h}$. (C) KRAS knockdown inhibited the expression of PDK2 and activation of PDHE1- $\alpha$. Cells were treated as in B. Ratio represents the intensity of band of pPDHE1- $\alpha$ normalized total PDHE1- $\alpha$ and then normalized by control. (D) Role of KRAS in the regulation of $\triangle \Psi \mathrm{m}$. PANC-1 cells were treated as indicated and $\triangle \Psi \mathrm{m}$ was observed using fluorescent microscope (left) and quantified (right). Cells with KRAS knockdown showed similar fluorescence compared with the control cells. (E) Knockdown of KRAS inhibited sphere formation in PANC-1 cells. Cells were treated as indicated and cultured as in the "Materials and Methods". (F) Effect of KRAS knockdown on Nanog and Sox2 expression in PANC-1 and SW1990 cells. Ratio is expressed as fold change compared with control values normalized to 1 after normalized by $\beta$-Actin. (G) PANC-1 and SW1990 cells were transfected with siRNA targeting KRAS as indicated. Analysis of qRT-PCR was used to examine the mRNA expression of Nanog and Sox2. (H) Effect of KRAS knockdown on cell viability. PANC-1 cells were plated in 96-well plates and transfected with siRNA targeting KRAS. After treatment, cells were further treated with increasing GEM (as indicated), 2-DG $(0,1,5,10,20,40,80 \mathrm{mM})$, or increasing GEM \& 2-DG (5 mM) for next $48 \mathrm{~h}$. Cell viability was analyzed using MTT assay and quantified with IC50. All experiments were repeated at least 3 times. Bars represent means \pm SD. $\beta$-Actin was served as an internal control. ${ }^{*} P<0.05 ;{ }^{* *} P<0.01$; ${ }^{* * *} P<0.001$. GEM: gemcitabine. 
glycolysis and decreased mitochondrial oxidation, accompanied by the enhanced cancer stemness. However, inhibition of glycolysis using 2-DG suppresses cancer stemness and strengthens the cytotoxicity of GEM, suggesting a rationale of combining glycolytic inhibitors with conventional chemotherapeutics for PanCa treatment.

Metabolic reprogramming has been recognized a core hallmark in cancer cells [37]. Oncogenic KRAS can lead to many of the phenotypic hallmarks of cancer including cellular metabolism [10]. KRAS transformation can promote glycolytic activity and decreased oxidative flux [38]. Ectopic $K R A S$ expression could inhibit mitochondrial respiratory chain activity, leading to the suppression of mitochondrial respiration [12]. Viale et al. found that surviving cells cultured in semisolid medium with KRAS ablation exhibited decreased glycolysis and relied on mitochondrial oxidation for survival [39]. Our study shows that GEM induces
A
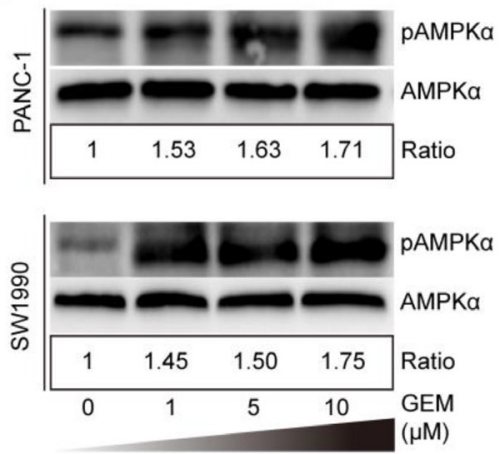

D

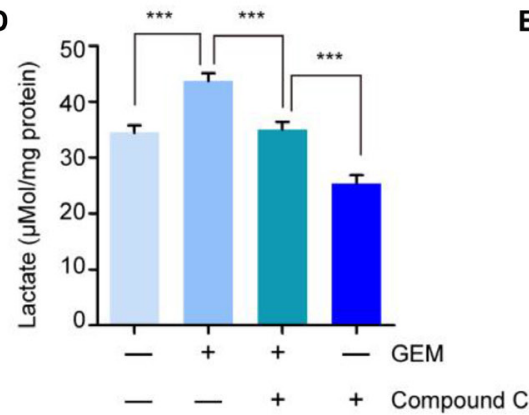

B

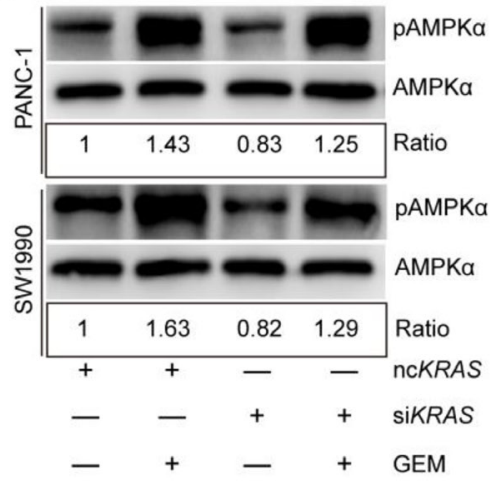

C

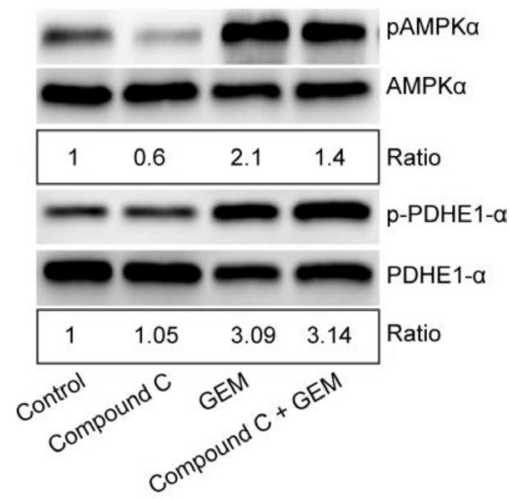

E

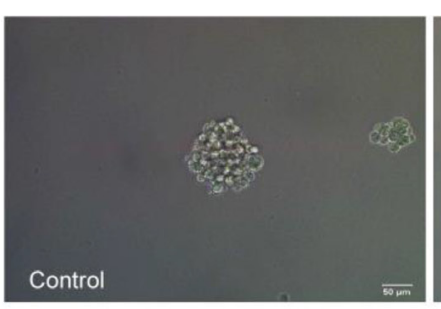

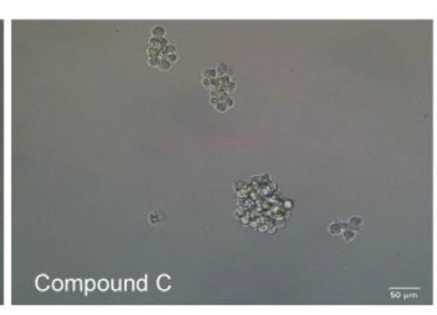

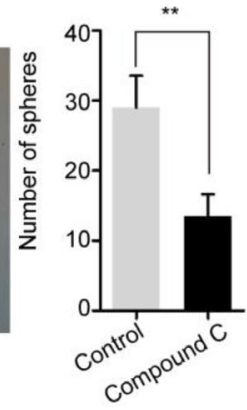

F

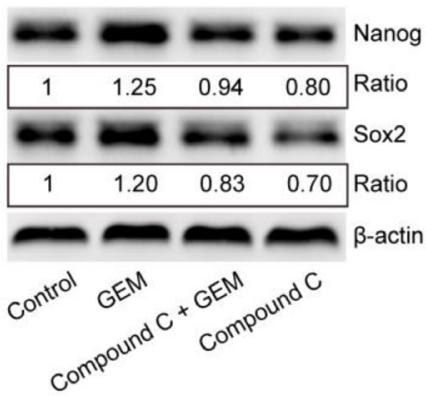

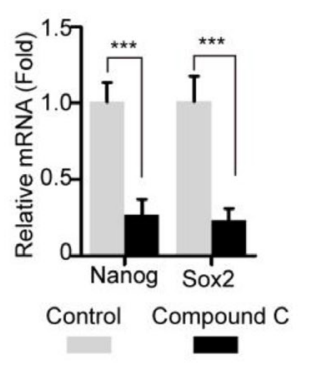

G

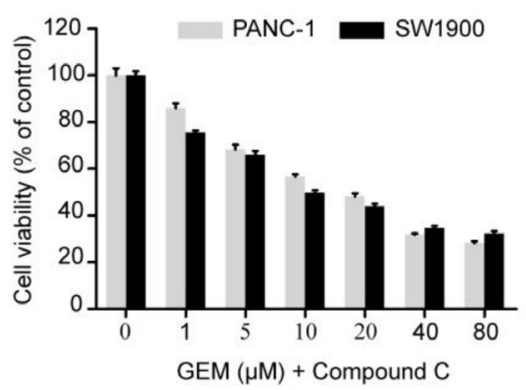

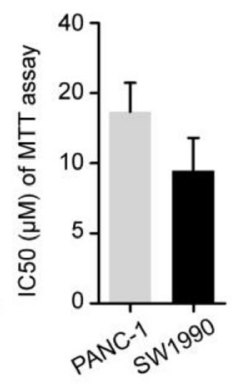

Figure 4: GEM-induced metabolic shift activates AMPK. (A) AMPK activation was examined by Western blot in PANC-1 and SW1990 cells treated as indicated. (B) GEM-induced activation of AMPK was reversed by KRAS knockdown. PANC-1 and SW1990 cells were treated as in Figure 3C. (C) Effect of AMPK inhibitor compound C on pAMPK $\alpha$ and p-PDHE1- $\alpha$. PANC-1 cells were pretreated with $1 \mu \mathrm{M}$ compound $\mathrm{C}$ for $1 \mathrm{~h}$ followed by treatment with $5 \mu \mathrm{M}$ GEM for $24 \mathrm{~h}$. (D) Compound C inhibited GEM-induced lactate production. PANC-1 cells were pretreated with $1 \mu \mathrm{M}$ compound $\mathrm{C}$ for $1 \mathrm{~h}$ followed by treatment with GEM $(5 \mu \mathrm{M})$ for $36 \mathrm{~h}$. (E) Compound C inhibited sphere formation ability in PANC-1 cells. PANC- 1 cells were treated with compound C $(1 \mu \mathrm{M}, 24 \mathrm{~h})$. The treated cells $\left(1 \times 10^{4}\right.$ cells) were cultured as indicated for sphere formation. Representative results (left) and quantification of the assay (right). (F) Compound $\mathrm{C}$ suppressed the expression of Nanog, Sox 2 in both protein and gene expressions. PANC-1 cells were treated as in C for Western blot or treated as in E and for qRT-PCR analysis. Ratio was consistent with previous description. (G) PANC-1 and SW1990 cells were treated with increasing concentration of GEM combined with compound C $(1 \mu \mathrm{M})$ for $48 \mathrm{~h}$. MTT assay were used to assess cell viability. Representative results (left) and quantification of IC50 (right). $\beta$-Actin was served as an internal control. All experiments were repeated at least 3 times. Bars represent means \pm SD. Ratio represents the intensity of band of pAMPK $\alpha$ or p-PDHE1- $\alpha$ normalized total AMPK $\alpha$ or PDHE1- $\alpha$, respectively, and then normalized by control. ${ }^{* *} P<0.01 ;{ }^{* * *} P<0.001$. GEM: gemcitabine. 
KRAS activation which in turn regulates metabolic reprogramming, as knockdown of $K R A S$ can inhibit glycolysis flux and restore mitochondria respiration. The restored cellular metabolism after KRAS knockdown suppresses cancer cell stemness, and sensitizes PanCa cells to GEM, 2-DG, or both, suggesting a tumorigenic and chemoresistant role for $K R A S$. Similarly, activation of mitochondrial oxidation by PDK2 inhibition could activate mitochondrial apoptotic signaling, causing the death of chemoresistant cancer cells [40]. Mitochondrial
OXPHOS was essential for efficient execution of cell death $[41,42]$. We did not observe the inhibitory effect of $K R A S$ knockdown on cancer cell viability, which might due to limited efficiency of siRNA-mediated silencing. The oncogenic role of mutant KRAS prompts intensive efforts to explore the pharmacological approaches. However, the pursued anti-KRAS therapies have not been successful in clinic practice. The connection between oncogenic $K R A S$ and metabolic reprogramming might provide promising directions [10].

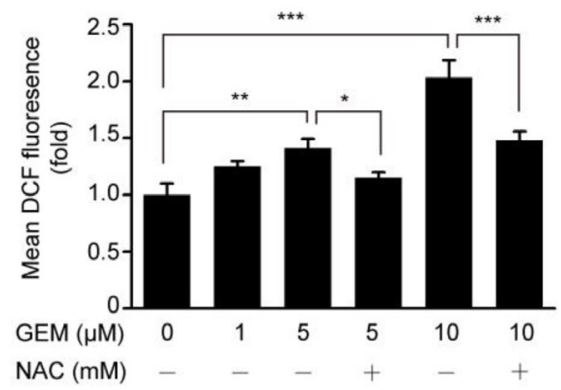

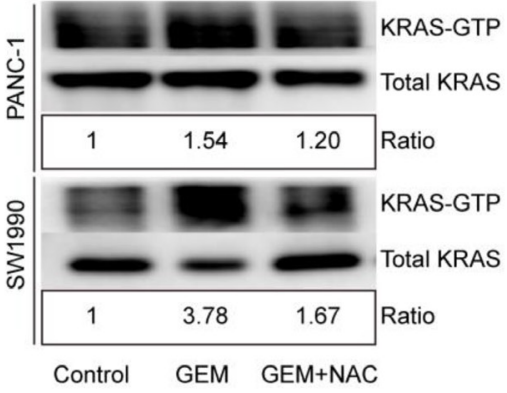

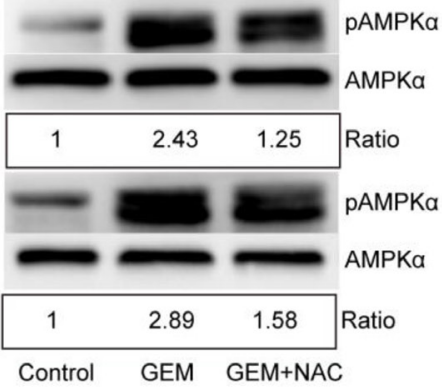

E

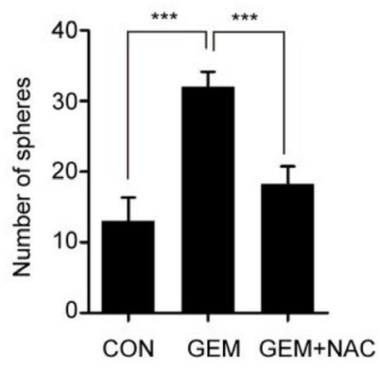

C
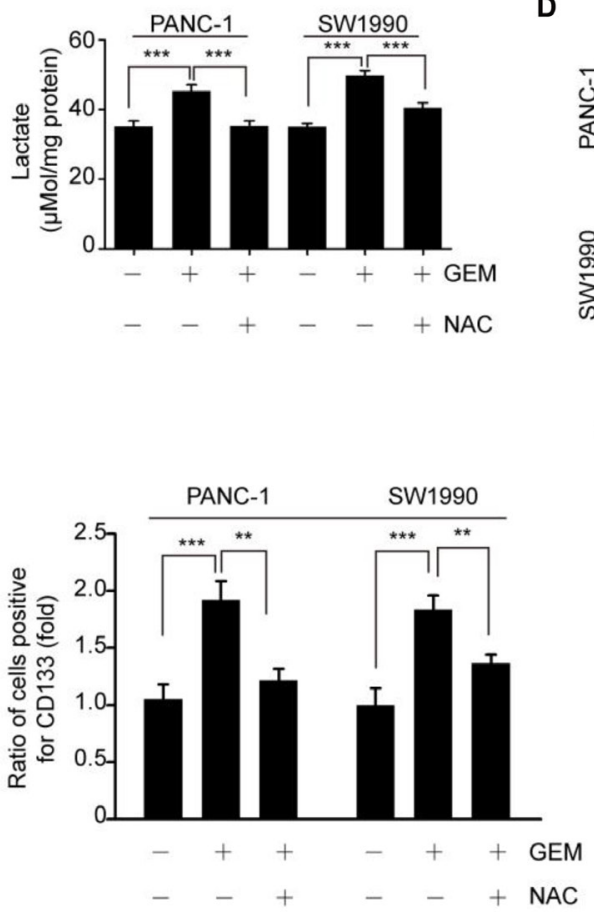

D
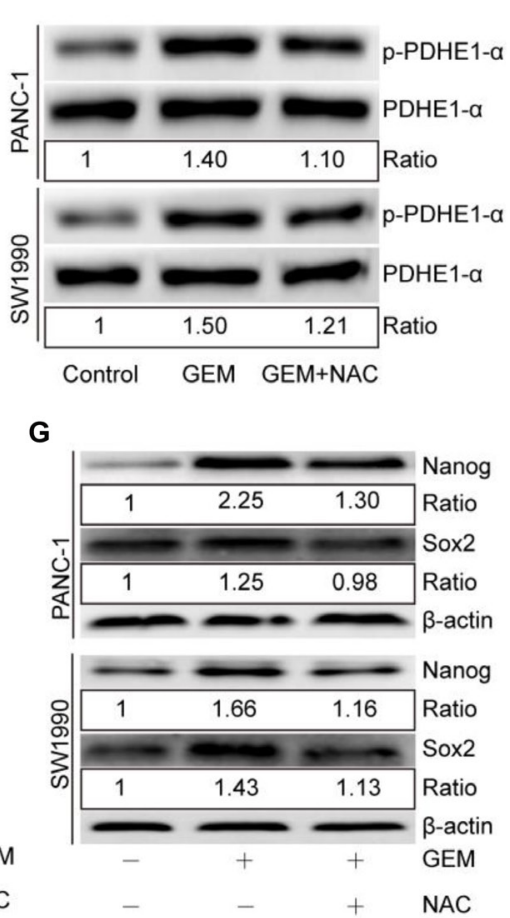

H

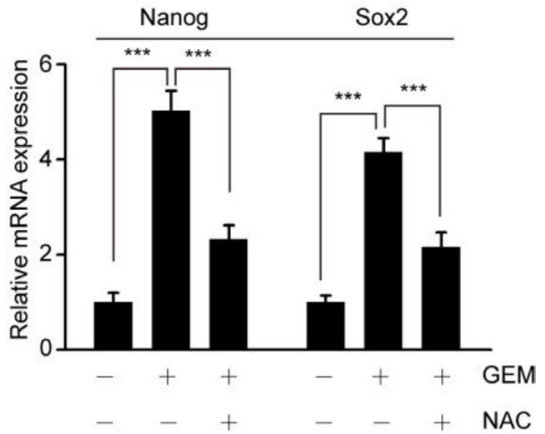

Figure 5: GEM-induced ROS is involved in the activation of KRAS/AMPK pathway. (A) ROS levels in PANC-1 cells pretreated with $5 \mathrm{mM}$ NAC (a ROS scavenger) for $1 \mathrm{~h}$ followed by treatment with different doses of GEM for $24 \mathrm{~h}$ were measured using flow cytometry with a DCFH-DA probe. (B) Role of ROS in the activation of KRAS/AMPK pathway. PANC-1 and SW1990 cells were pretreated with NAC $(5 \mathrm{mM}, 1 \mathrm{~h})$ followed by treatment with GEM $(5 \mu \mathrm{M})$ for $24 \mathrm{~h}$. Activation of KRAS and AMPK was measured by Western blot. (C) Lactate production in PANC-1 and SW1990 cells pretreated with NAC $(5 \mathrm{mM}, 1 \mathrm{~h})$ followed by treatment with GEM $(5 \mu \mathrm{M})$ for $36 \mathrm{~h}$. (D) Levels of p-PDHE1- $\alpha$ in cells treated as in B were examined by Western blot. Ratio represents the intensity of band of p-PDHE1- $\alpha$ normalized total PDHE1- $\alpha$ and then normalized by control. (E-H) Effect of GEM-induced ROS on cancer stemness. (E) PANC-1 cells were treated as in (B). The treated cells were cultured as described. (F-G) CD133 and pluripotent markers Nanog and Sox2 were examined using flow cytometry or Western blot in cells treated as in (B). Ratio was consistent with previous description. (H) Analysis of qRT-PCR was used to examine the mRNA level of Nanog and Sox 2 in PANC-1 cells treated as in (B). $\beta$-Actin was served as an internal control. All experiments were repeated at least 3 times. Bars represent means \pm SD. Ratio represents the intensity of band of KRAS-GTP, or pAMPK $\alpha$ normalized total KRAS or AMPK $\alpha$ and then normalized by control. ${ }^{*} P<0.05 ;{ }^{* *} P<0.01$; ${ }^{* * *} P<0.001$. GEM: gemcitabine. 
It was reported that GEM-induced ROS was generated by activation of NADPH oxidase (NOX) through NF- $\kappa$ B activation as siRNA depletion of $\mathrm{p} 22^{\text {-phox }}$ (a catalytic subunit of the NOX complex) alleviated GEM-induced ROS production [17]. We also found that GEM-induced ROS was NOX-dependent as inhibition of NOX using Apocynin (a NOX inhibitor) reduced ROS production [43]. ROS-induced activation of NF- $\kappa \mathrm{B}$ can also promote HIF$1 \alpha$ and CXCR4 expression, which was involved in GEM resistance in PanCa cells [18]. Our findings show that
GEM-induced ROS participates in the activation of KRAS/ AMPK pathway, which can be reversed by pre-treatment with NAC, a free radical scavenger. It was reported that CSCs have mechanisms to fine-tune ROS levels by tightly regulating metabolic pathways such as glycolysis rather than oxidative phosphorylation to reduce oxidative stress [44]. Nomura et al. found that $\mathrm{CD} 133^{+}$pancreatic CSCs showed increased glycolytic flux and decreased mitochondrial activity, and the metabolic switch resulted in decreased ROS production upon GEM treatment and favored their
A

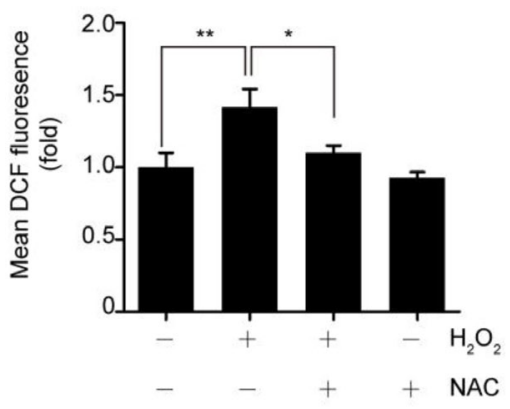

C

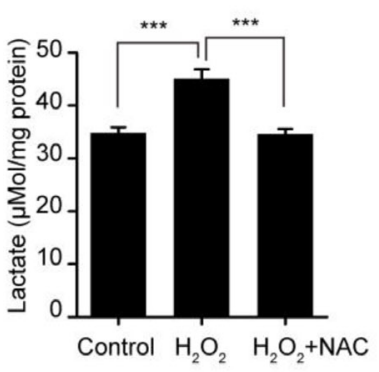

$\mathbf{F}$

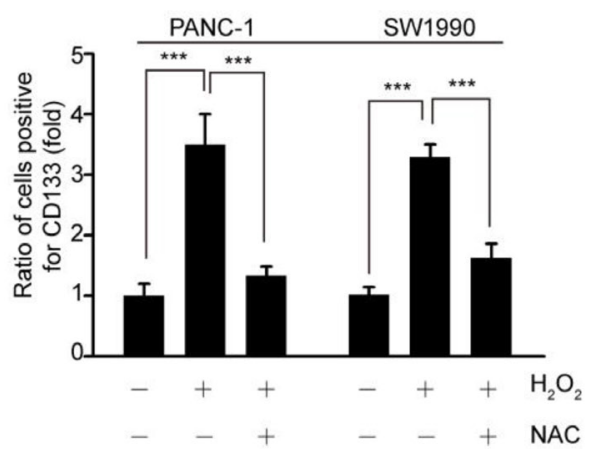

B

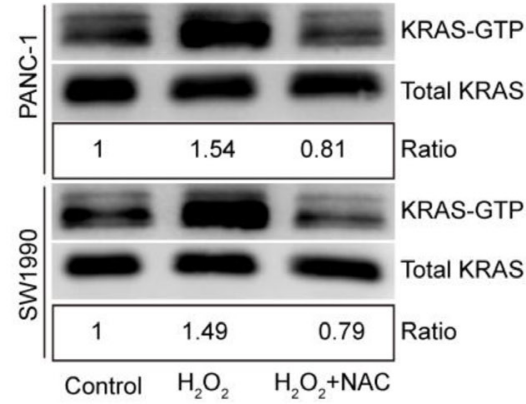

D

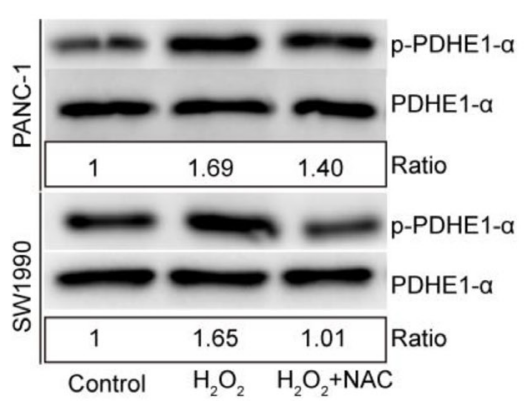

G

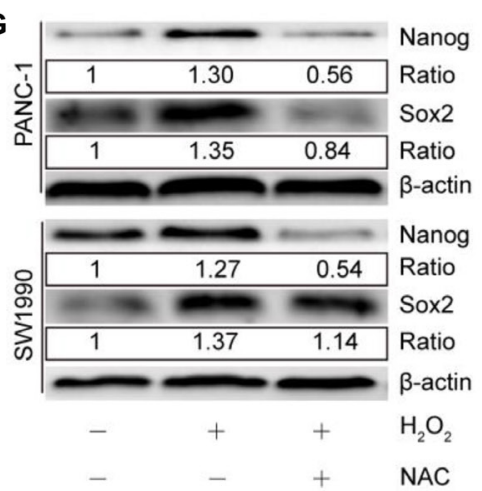

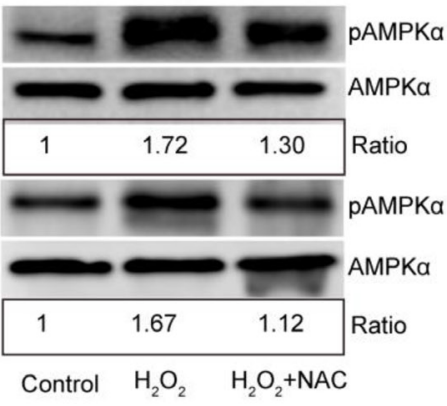

$\mathbf{E}$

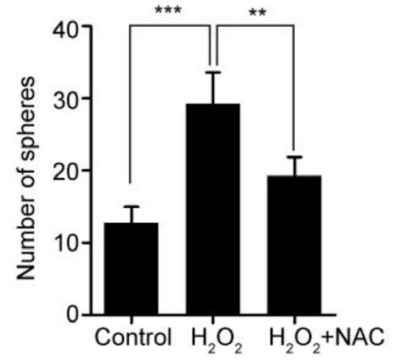

H

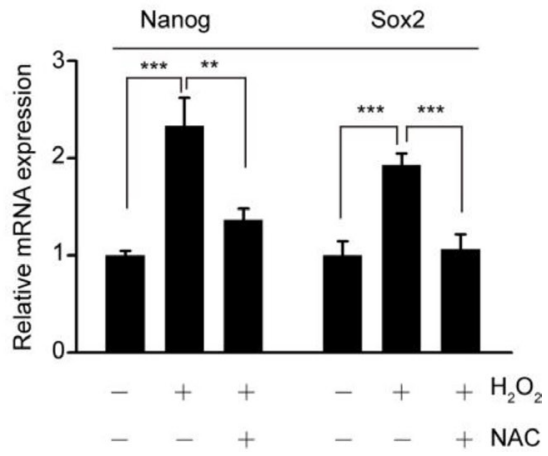

Figure 6: The verification of ROS in activation of KRAS/AMPK pathway. (A) ROS levels in PANC-1 cells pretreated with NAC $(5 \mathrm{mM}, 1 \mathrm{~h})$ followed by treatment with $\mathrm{H}_{2} \mathrm{O}_{2}(200 \mu \mathrm{M})$ for $12 \mathrm{~h}$ were assessed using flow cytometry. (B) The activation of KRAS/ AMPK was assessed by Western blot. PANC-1 and SW1990 cells were pretreated with NAC $(5 \mathrm{mM}, 1 \mathrm{~h})$ followed by treatment with $\mathrm{H}_{2} \mathrm{O}_{2}$ $(200 \mu \mathrm{M})$ for $24 \mathrm{~h}$. (C) Lactate production in PANC-1 cells pretreated with NAC $(5 \mathrm{mM}, 1 \mathrm{~h})$ followed by treatment with $\mathrm{H}_{2} \mathrm{O}_{2}(200 \mu \mathrm{M})$ for $36 \mathrm{~h}$. (D) Levels of p-PDHE1- $\alpha$ in cells treated as in B were examined by Western blot. (E-H) $\mathrm{H}_{2} \mathrm{O}_{2}$-induced ROS were involved in the induction of cancer stemness status. Cells were treated as in (B, E) Sphere formation assay of PANC-1 cells were shown. (F-G) CD133 and pluripotent markers Nanog and Sox2 were examined using flow cytometry or Western blot in PANC-1 and SW1990 cells treated as in (B, H). The mRNA expressions of Nanog and Sox 2 in PANC-1 cells treated as in B. $\beta$-Actin was served as an internal control. All experiments were repeated at least 3 times. Bars represent means $\pm \mathrm{SD}$. Ratio was consistent with previous description. ${ }^{*} P<0.05$; ${ }^{* *} P<0.01 ;{ }^{* * *} P<0.001$. GEM: gemcitabine. 
survival [30]. Thus, the metabolic switch induced by GEM was likely a cellular response to oxidative stress and prerequisite for the induction of CSCs. In addition, GEMinduced expression of the antioxidant genes may also play a role in the maintenance of intracellular redox homeostasis. GEM treatment increased Nrf2 expression which regulated the expression of glutathione (GSH), a major cellular antioxidant, to maintain ROS levels within threshold in case of oxidative damage [17]. Additionally, ROS-dependent regulation of energy metabolism can drive the carbohydrate flux to pentose phosphate pathway, thereby increasing the NADPH level to counteract intracellular ROS [45]. Furthermore, fatty acid oxidation key enzyme carnitine palmitoyltransferase 1A (CPT-1A) maintains intracellular redox and energy homeostasis via generation of NADPH and GSH during energy stress [46]. Cancer cells can rewire metabolic pathways in response to changes in cellular energy and nutrient status, suggesting the complexity of targeting cellular metabolism.

AMPK is an conserved energy sensor and master regulator of cellular metabolism [31]. We found that GEM treatment activates AMPK, probably resulting from the energy crisis induced by GEM treatment as the restoration of mitochondria oxidation after KRAS knockdown inhibits AMPK activation. Interestingly, AMPK-dependent activator promotes glycolysis while AMPK inhibitor inhibits glycolysis flux, suggesting a direct regulatory role of AMPK in glycolysis, which was consistent with recent findings [33]. AMPK activation may also promote intracellular NADPH production against oxidative stress under energy stress conditions [33, 47]. Besides, AMPK was critical in modulating the self-renewal and chemoresistance of CSCs [15]. We find that AMPK activation promoted glycolysis, cancer cell stemness and survival, suggesting AMPK as a potential target against the malignant behavior of PanCa.

In conclusion, we demonstrate an undesired effect of GEM in PanCa treatment. GEM induces KRAS activation, which contributes to metabolic reprogramming from mitochondrial oxidation to glycolysis and enhanced cancer cell stemness. The metabolic shift activates AMPK which further promotes glycolysis and enhances cancer cell pluripotency. Furthermore, GEMinduced ROS participates in the activation of KRAS/ AMPK signaling, metabolic reprogramming and cancer stemness maintenance. Considering that metabolic reprograming was implicated in pluripotent redirection and the contributing role of glycolysis in chemoresistance, interventions targeting cellular metabolism might provide new therapeutic opportunities to PanCa treatments.

\section{MATERIALS AND METHODS}

\section{Reagents}

Stock solutions of GEM (Selleck.cn, Shanghai, China) were made every week in $0.9 \%$ normal saline. 2-DG (Sigma-Aldrich Co., St. Louis, MO, USA) was freshly prepared by dissolving powder into serum-free medium as needed. Hydrogen peroxide $\left(\mathrm{H}_{2} \mathrm{O}_{2}\right)$ (Amresco, USA) was freshly made by dissolving the original solution in normal saline. N-acetylcysteine (NAC) (Amresco, USA) was also dissolved in normal saline. Compound C and A-769662 were purchased form Sellect Chemicals

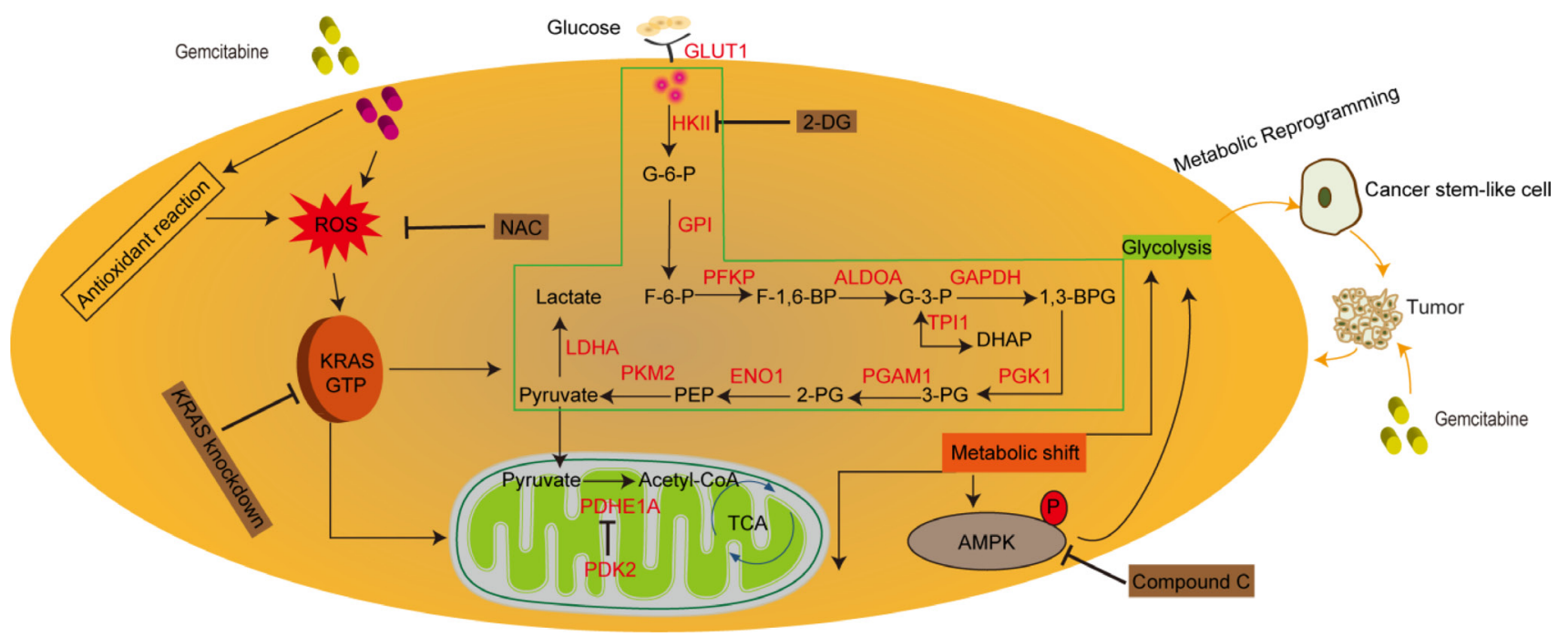

Figure 7: GEM-induced activation of ROS-KRAS-AMPK-signaling axis regulates PanCa cell metabolism and cancer cell stemness. GEM treatment induces ROS-mediated, KRAS-dependent metabolic reprogramming from mitochondrial oxidation to glycolysis, leading to the induction of cancer stem-like cells, accounting for chemoresistance and tumor recurrence. The metabolic shift induced by GEM activates AMPK which further promotes glycolysis and cancer stemness. However, ROS scavenger (NAC), KRAS knockdown, AMPK inhibitor (Compound C), or glycolytic inhibitor (2-DG) can inhibit GEM-induced cancer cell stemness. Abbreviation: see Supplementary Figure 1A. GEM: gemcitabine. 
(Houston, TX) and was dissolved in DMSO according to the manufacturer's instruction.

\section{Cell lines and culture conditions}

The human PanCa cell lines PANC-1 and SW1990 were originally obtained from American Type Culture Collection (ATCC) (Manassas, USA). Patu8988 cell line was obtained from Keygen (Key Gen Bio Tech, China). All three cell lines have point mutation at codon 12 of KRAS gene (GGT $\rightarrow$ GAT in PANC-1 and SW1990 and GGT $\rightarrow$ GTT in Patu8988). The cells were maintained in culture media recommended. Media were supplemented with $1 \%$ penicillin/streptomycin and $10 \%$ fetal bovine serum (Gibco Invitrogen, Grand Island, NY, USA). The cells were maintained in water-saturated air with $5 \% \mathrm{CO}_{2}$ at $37^{\circ} \mathrm{C}$.

\section{Cell viability assay}

The viability was analyzed using a 3-(4, 5-dimethyl2-thiazolyl)-2, 5-diphenyl-2H-tetrazolium bromide (MTT) assay (Sigma-Aldrich). PanCa cells $(6,000 /$ well) were seeded in 96-well plates overnight. The cells were then treated with increasing concentrations of GEM $(1 \mu \mathrm{M}$ to $80 \mu \mathrm{M})$ combined with or without 2-DG $(5 \mathrm{mM})$ for $48 \mathrm{~h}$. PANC-1 cells $\left(3 \times 10^{3}\right)$ were seeded and transfected with siRNA targeting $K R A S$ (siKRAS) or negative control siRNA (ncKRAS) for $48 \mathrm{~h}$ and then treated with GEM $(5 \mu \mathrm{M}), 2-\mathrm{DG}(5 \mathrm{mM})$, or their combination for another $36 \mathrm{~h}$. Next, $20 \mu \mathrm{L}$ of MTT $(5 \mathrm{mg} / \mathrm{mL}$ in phosphate-buffered saline [PBS]) was added and incubated for another $4 \mathrm{~h}$. After that, the supernatant of each well was discarded and dimethyl sulfoxide (DMSO) (Sigma-Aldrich) $(150 \mu \mathrm{L})$ was added. Following agitation for $10 \mathrm{~min}$ in the dark on an Eppendorf shaker, absorbance was read at $490 \mathrm{~nm}$ in a microplate photometer. Each concentration was tested in 5 replicates. Data were expressed relative to the untreated group.

\section{Western blot analysis}

Cells were lysed with a radioimmunoprecipitation assay lysis buffer (Beyotime Biotechnology, Shanghai, China) at $4^{\circ} \mathrm{C}$. Equal amounts of protein were subjected to $10 \%$ SDS-PAGE and transferred to PVDF membranes (Millipore, Billerica, MA, USA) which were then blocked with $5 \%$ non-fat for $1 \mathrm{~h}$ and incubated with primary antibodies over night at $4^{\circ} \mathrm{C}$. The membranes were washed and incubated with secondary horseradish peroxidase-coupled antibodies (Aspen, Wuhan, China), and visualized using ECL substrate (Thermo Fisher, Waltham, MA, USA). Primary antibodies against Nanog, Sox2, phospho-AMPK $\alpha$, and AMPK $\alpha$ were acquired from Cell Signaling Technologies (MA, USA). The antibodies against GLUT1 and LDHA were bought form Cusa Bio (Wuhan, China). The antibodies against $\beta$-Actin and
KRAS were purchased from ProteinTech (Wuhan, China). The antibodies against PDK2, phospho-PDHE1- $\alpha$ and PDHE1- $\alpha$ were obtained from Abcam (Cambridge, MA). Image Lab software (Version 3.0) (Bio-Rad Lab, Inc, Hercules, CA) was used to quantify the band density.

\section{Quantitative real-time PCR assay}

Total RNA was extracted using TRIzol (Invitrogen). Equal amount of RNA $(0.5 \mu \mathrm{g})$ was reverse-transcribed to cDNA using PrimeScript RT Master Mix (Takara Bio). The qRT-PCR analysis was performed using a quantitative SYBR Green PCR Kit (Takara Bio) according to the manufacturer's instruction. The data were interpreted with the $2^{-\Delta \Delta C T}$ method and normalized to $\beta$-Actin. Primer sequences are shown in Supplementary Table 1.

\section{Flow cytometric analysis}

Cells were dissociated into single cells and washed with PBS followed by stained with PE-conjugated CD133 antibody or isotype control IgG (Biolegend, San Diego, $\mathrm{CA}, \mathrm{USA})$ and were analyzed using a flow cytometer (BD, Biosciences, USA).

\section{Determination of intracellular ROS levels and mitochondria membrane potential $(\Delta \Psi \mathrm{m})$}

Cells were treated as indicated and incubated with dichloro-dihydro-fluorescein diacetate (DCFH-DA) (Beyotime) at a final concentration of $10 \mu \mathrm{M}$ in serumfree medium for $20 \mathrm{~min}$ at $37^{\circ} \mathrm{C} . \Delta \Psi \mathrm{m}$ of PanCa cells was measured using mitochondrial membrane potential assay kit with JC-1 (Beyotime) according to the manufacturer's instruction. The fluorescence was then measured with a flow cytometry or fluorescent microscope.

\section{Sphere-formation assay}

Cells $\left(5-10 \times 10^{3}\right)$ with different treatments were cultured in serum-free Dulbecco's modified Eagle medium (DMEM)/Ham's nutrient mixture (F12) (1:1) medium (Invitrogen) supplemented with $20 \mathrm{ng} / \mathrm{mL}$ of epithelial growth factor and $10 \mathrm{ng} / \mathrm{mL}$ of basic fibroblast growth factor (PeproTech) for 2 weeks. Spheres larger than $50 \mu \mathrm{m}$ were counted using a microscope (Olympus, Tokyo, Japan).

\section{Small interfering RNA-mediated knockdown of KRAS}

Small interfering RNA (siRNA) sequence targeting the coding region of KRAS (siKRAS: GGAAG CAAGTAATTGA) and negative control siRNA (ncKRAS) not matching any of the human genes were obtained (RiboBio Co. Guangzhou, China). Knockdown of KRAS was performed by transfecting cells with Lipofectamine 2000 (Invitrogen) according to the manufacturer's 
instructions. Efficiency of the transfection was confirmed by Western blot and qRT-PCR analyses.

\section{Glucose uptake and lactate production assays}

Glucose uptake was performed according to previously published methods [48]. For assessment of lactate production, cells $\left(3 \times 10^{5}\right)$ were cultured overnight. After different treatments, the supernant was collected and centrifuged. Lactate production was assessed using a lactic acid assay kit (Nanjing Jiancheng Bio. Nanjing, China) according to the manufacturer's protocol and was corrected for the total protein in each sample.

\section{KRAS activation assay}

The level of active KRAS was assessed utilizing a RAS activation assay kit (NewEast Biosciences, Malvern, PA). Briefly, cells were cultured in $10 \mathrm{~cm}$ plated to $80-90 \%$ confluence before different treatments. Total cell lysates were collected and incubated with anti-active RAS monoclonal antibody plus protein $\mathrm{A} / \mathrm{G}$ agarose bead slurry at $4^{\circ} \mathrm{C}$ for $1 \mathrm{~h}$ with gentle agitation. The agarose beads were resuspended in $20 \mu \mathrm{L} 2 \times$ reducing SDS-PAGE sample buffer and boiled. The precipitated active RAS was detected by immunoblot analysis using anti-KRAS monoclonal antibody according to the manufacturer's instructions.

\section{Statistical analysis}

All statistical analyses were performed by GraphPad Prism 5.0 software (San Diego, CA). Unpaired $t$ tests, one-way ANOVA with Bonferroni post-tests, or two-way ANOVA were used to calculate statistical significance. Results were presented as means \pm standard deviation (SD). $P$ values $<0.05$ were considered statistically significant.

\section{ACKNOWLEDGMENTS}

This work was supported by the National Natural Science Foundation of China (No. 81372665 to T Y.). The authors thank Amy Ninetto, PhD, ELS, Department of Scientific Publications, MD Anderson Cancer Center, for her editing of the manuscript.

\section{CONFLICTS OF INTEREST}

The authors declare that there is no conflicts of interests regarding the publication of this paper.

\section{REFERENCES}

1. de Sousa Cavalcante L, Monteiro G. Gemcitabine: metabolism and molecular mechanisms of action, sensitivity and chemoresistance in pancreatic cancer. Eur J Pharmacol. 2014; 741:8-16.

2. Gangemi R, Paleari L, Orengo AM, Cesario A, Chessa L, Ferrini S, Russo P. Cancer stem cells: a new paradigm for understanding tumor growth and progression and drug resistance. Curr Med Chem. 2009; 16:1688-1703.

3. Du Z, Qin R, Wei C, Wang M, Shi C, Tian R, Peng C. Pancreatic cancer cells resistant to chemoradiotherapy rich in "stem-cell-like" tumor cells. Dig Dis Sci. 2011; $56: 741-750$

4. Lee CJ, Dosch J, Simeone DM. Pancreatic cancer stem cells. J Clin Oncol. 2008; 26:2806-2812.

5. Lee HJ, You DD, Choi DW, Choi YS, Kim SJ, Won YS, Moon HJ. Significance of CD133 as a cancer stem cell markers focusing on the tumorigenicity of pancreatic cancer cell lines. J Korean Surg Soc. 2011; 81:263-270.

6. Deshmukh A, Deshpande K, Arfuso F, Newsholme P, Dharmarajan A. Cancer stem cell metabolism: a potential target for cancer therapy. Mol Cancer. 2016; 15:69.

7. Liang C, Qin Y, Zhang B, Ji S, Shi S, Xu W, Liu J, Xiang J, Liang D, Hu Q, Ni Q, Xu J, Yu X. Metabolic plasticity in heterogeneous pancreatic ductal adenocarcinoma. Biochim Biophys Acta. 2016; 1866:177-188.

8. Lee SY, Jeong EK, Ju MK, Jeon HM, Kim MY, Kim CH, Park HG, Han SI, Kang HS. Induction of metastasis, cancer stem cell phenotype, and oncogenic metabolism in cancer cells by ionizing radiation. Mol Cancer. 2017; 16:10.

9. Kim RK, Cui YH, Yoo KC, Kim IG, Lee M, Choi YH, Suh Y, Lee SJ. Radiation promotes malignant phenotypes through SRC in breast cancer cells. Cancer Sci. 2015; 106:78-85.

10. Bryant KL, Mancias JD, Kimmelman AC, Der CJ. KRAS: feeding pancreatic cancer proliferation. Trends Biochem Sci. 2014; 39:91-100.

11. Levine AJ, Puzio-Kuter AM. The control of the metabolic switch in cancers by oncogenes and tumor suppressor genes. Science. 2010; 330:1340-1344.

12. Hu Y, Lu W, Chen G, Wang $\mathrm{P}$, Chen Z, Zhou Y, Ogasawara M, Trachootham D, Feng L, Pelicano H, Chiao PJ, Keating MJ, Garcia-Manero G, et al. K-ras(G12V) transformation leads to mitochondrial dysfunction and a metabolic switch from oxidative phosphorylation to glycolysis. Cell Res. 2012; 22:399-412.

13. Folmes CD, Nelson TJ, Dzeja PP, Terzic A. Energy metabolism plasticity enables stemness programs. Ann N Y Acad Sci. 2012; 1254:82-89.

14. Xiao X, Su G, Brown SN, Chen L, Ren J, Zhao P. Peroxisome proliferator-activated receptors gamma and alpha agonists stimulate cardiac glucose uptake via activation of AMP-activated protein kinase. J Nutr Biochem. 2010; 21:621-626.

15. Wang Z, Wang N, Liu P, Xie X. AMPK and Cancer. EXS. 2016; 107:203-226.

16. Donadelli M, Costanzo C, Beghelli S, Scupoli MT, Dandrea M, Bonora A, Piacentini P, Budillon A, 
Caraglia M, Scarpa A, Palmieri M. Synergistic inhibition of pancreatic adenocarcinoma cell growth by trichostatin A and gemcitabine. Biochim Biophys Acta. 2007; 1773:1095-1106.

17. Ju HQ, Gocho T, Aguilar M, Wu M, Zhuang ZN, Fu J, Yanaga K, Huang P, Chiao PJ. Mechanisms of Overcoming Intrinsic Resistance to Gemcitabine in Pancreatic Ductal Adenocarcinoma through the Redox Modulation. Mol Cancer Ther. 2015; 14:788-798.

18. Arora S, Bhardwaj A, Singh S, Srivastava SK, McClellan S, Nirodi CS, Piazza GA, Grizzle WE, Owen LB, Singh AP. An undesired effect of chemotherapy: gemcitabine promotes pancreatic cancer cell invasiveness through reactive oxygen species-dependent, nuclear factor kappaBand hypoxia-inducible factor 1alpha-mediated up-regulation of CXCR4. J Biol Chem. 2013; 288:21197-21207.

19. Zhao H, Duan Q, Zhang Z, Li H, Wu H, Shen Q, Wang C, Yin T. Up-regulation of glycolysis promotes the stemness and EMT phenotypes in gemcitabine-resistant pancreatic cancer cells. J Cell Mol Med. 2017.

20. Kurtoglu M, Maher JC, Lampidis TJ. Differential toxic mechanisms of 2-deoxy-D-glucose versus 2-fluorodeoxyD-glucose in hypoxic and normoxic tumor cells. Antioxid Redox Signal. 2007; 9:1383-1390.

21. Bonnet S, Archer SL, Allalunis-Turner J, Haromy A, Beaulieu C, Thompson R, Lee CT, Lopaschuk GD, Puttagunta L, Bonnet S, Harry G, Hashimoto K, Porter CJ, et al. A mitochondria-K+ channel axis is suppressed in cancer and its normalization promotes apoptosis and inhibits cancer growth. Cancer Cell. 2007; 11:37-51.

22. Abel EV, Simeone DM. Biology and clinical applications of pancreatic cancer stem cells. Gastroenterology. 2013; 144:1241-1248.

23. Li X, Han G, Li X, Kan Q, Fan Z, Li Y, Ji Y, Zhao J, Zhang M, Grigalavicius M, Berge V, Goscinski MA, Nesland JM, et al. Mitochondrial pyruvate carrier function determines cell stemness and metabolic reprogramming in cancer cells. Oncotarget. 2017; 8:46363-46380.

24. Herreros-Villanueva M, Bujanda L, Billadeau DD, Zhang JS. Embryonic stem cell factors and pancreatic cancer. World J Gastroenterol. 2014; 20:2247-2254.

25. Hermann PC, Huber SL, Herrler T, Aicher A, Ellwart JW, Guba M, Bruns CJ, Heeschen C. Distinct populations of cancer stem cells determine tumor growth and metastatic activity in human pancreatic cancer. Cell Stem Cell. 2007; 1:313-323.

26. Modok S, Mellor HR, Callaghan R. Modulation of multidrug resistance efflux pump activity to overcome chemoresistance in cancer. Curr Opin Pharmacol. 2006; 6:350-354.

27. Maruyama H, Kleeff J, Wildi S, Friess H, Buchler MW, Israel MA, Korc M. Id-1 and Id-2 are overexpressed in pancreatic cancer and in dysplastic lesions in chronic pancreatitis. Am J Pathol. 1999; 155:815-822.
28. Lappi-Blanco E, Makinen JM, Lehtonen S, Karvonen H, Sormunen R, Laitakari K, Johnson S, Makitaro R, Bloigu R, Kaarteenaho R. Mucin-1 correlates with survival, smoking status, and growth patterns in lung adenocarcinoma. Tumour Biol. 2016; 37:13811-13820.

29. Nomura A, Banerjee S, Chugh R, Dudeja V, Yamamoto M, Vickers SM, Saluja AK. CD133 initiates tumors, induces epithelial-mesenchymal transition and increases metastasis in pancreatic cancer. Oncotarget. 2015; 6:8313-8322.

30. Nomura A, Dauer P, Gupta V, McGinn O, Arora N, Majumdar K, Uhlrich C, 3rd, Dalluge J, Dudeja V, Saluja A, Banerjee S. Microenvironment mediated alterations to metabolic pathways confer increased chemo-resistance in CD133+ tumor initiating cells. Oncotarget. 2016; 7:56324-56337.

31. Long YC, Zierath JR. AMP-activated protein kinase signaling in metabolic regulation. J Clin Invest. 2006; 116:1776-1783.

32. Hawley SA, Davison M, Woods A, Davies SP, Beri RK, Carling D, Hardie DG. Characterization of the AMPactivated protein kinase kinase from rat liver and identification of threonine 172 as the major site at which it phosphorylates AMP-activated protein kinase. J Biol Chem. 1996; 271:27879-27887.

33. Wu SB, Wei YH. AMPK-mediated increase of glycolysis as an adaptive response to oxidative stress in human cells: implication of the cell survival in mitochondrial diseases. Biochim Biophys Acta. 2012; 1822:233-247.

34. Warsame R, Grothey A. Treatment options for advanced pancreatic cancer: a review. Expert Rev Anticancer Ther. 2012; 12:1327-1336.

35. Yang M, Liu P, Huang P. Cancer stem cells, metabolism, and therapeutic significance. Tumour Biol. 2016; 37:5735-5742.

36. Schieber MS, Chandel NS. ROS links glucose metabolism to breast cancer stem cell and EMT phenotype. Cancer Cell. 2013; 23:265-267.

37. Hanahan D, Weinberg RA. Hallmarks of cancer: the next generation. Cell. 2011; 144:646-674.

38. Gaglio D, Metallo CM, Gameiro PA, Hiller K, Danna LS, Balestrieri C, Alberghina L, Stephanopoulos G, Chiaradonna F. Oncogenic K-Ras decouples glucose and glutamine metabolism to support cancer cell growth. Mol Syst Biol. 2011; 7:523.

39. Viale A, Pettazzoni P, Lyssiotis CA, Ying H, Sanchez N, Marchesini M, Carugo A, Green T, Seth S, Giuliani V, KostAlimova M, Muller F, Colla S, et al. Oncogene ablationresistant pancreatic cancer cells depend on mitochondrial function. Nature. 2014; 514:628-632.

40. Roh JL, Park JY, Kim EH, Jang HJ, Kwon M. Activation of mitochondrial oxidation by PDK2 inhibition reverses cisplatin resistance in head and neck cancer. Cancer Lett. 2016; 371:20-29. 
41. Dey R, Moraes CT. Lack of oxidative phosphorylation and low mitochondrial membrane potential decrease susceptibility to apoptosis and do not modulate the protective effect of $\mathrm{Bcl}-\mathrm{x}(\mathrm{L})$ in osteosarcoma cells. J Biol Chem. 2000; 275:7087-7094.

42. Tomiyama A, Serizawa S, Tachibana K, Sakurada K, Samejima H, Kuchino Y, Kitanaka C. Critical role for mitochondrial oxidative phosphorylation in the activation of tumor suppressors Bax and Bak. J Natl Cancer Inst. 2006; 98:1462-1473.

43. Zhang Z, Duan Q, Zhao H, Liu T, Wu H, Shen Q, Wang C, Yin T. Gemcitabine treatment promotes pancreatic cancer stemness through the Nox/ROS/NF-kappaB/STAT3 signaling cascade. Cancer Lett. 2016; 382:53-63.

44. Ushio-Fukai M, Rehman J. Redox and metabolic regulation of stem/progenitor cells and their niche. Antioxid Redox Signal. 2014; 21:1587-1590.
45. Kang SW, Lee S, Lee EK. ROS and energy metabolism in cancer cells: alliance for fast growth. Arch Pharm Res. 2015; 38:338-345.

46. Luo J, Hong Y, Tao X, Wei X, Zhang L, Li Q. An indispensable role of CPT-1a to survive cancer cells during energy stress through rewiring cancer metabolism. Tumour Biol. 2016.

47. Jeon SM, Chandel NS, Hay N. AMPK regulates NADPH homeostasis to promote tumour cell survival during energy stress. Nature. 2012; 485:661-665.

48. Sharma BK, Kolhe R, Black SM, Keller JR, Mivechi NF, Satyanarayana A. Inhibitor of differentiation 1 transcription factor promotes metabolic reprogramming in hepatocellular carcinoma cells. FASEB J. 2016; 30:262-275. 